\title{
ON FORMAL THEORIES
}

\author{
KATUZI ONO
}

\section{Introduction}

The main purpose of the present paper is to introduce a new understanding of formal theories.

It has been a traditional pattern of formal theories to presuppose a logic and an axiom system for each formal theory. The axiom system of any formal theory consists of a finite number of axiom schemata in general, but occasionally it can be regarded as consisting of a finite number of axioms. I will call any formal theory of this kind an axiomatic theory or an axiom-schematic theory according as its axiom system is regarded as consisting of a finite number of axioms or as consisting of a finite number of axiom schemata.

Any axiomatic theory or any axiom-schematic theory has its own logic on which it stands. Possibly, we can establish various axiomatic theories or axiom-schematic theories standing on various logics even when we start from the same axiom system.

In my papers [1] and [2], I have pointed out that $\boldsymbol{J}$-series logics (the intuitionistic logic $\boldsymbol{L} \boldsymbol{J}$, the minimal logic $\boldsymbol{L M}$, and the positive logic $\boldsymbol{L} \boldsymbol{P}$, each without admitting the Peirce rule) as well as $\boldsymbol{K}$-series logics (the classical logic $\boldsymbol{L} \boldsymbol{K}$, the minimal logic $\boldsymbol{L} \boldsymbol{N}$, and the positive logic $\boldsymbol{L Q}$, each admitting the Peirce rule) can be faithfully interpreted in the primitive logic $\boldsymbol{L O}$ (the sub-logic of the intuitionistic logic $\boldsymbol{L} \boldsymbol{J}$ having "implication" and "universal quantification" only as its logical constants). This seems to suggest that there is a basic logic behind seemingly various logics of formal theories.

Any axiom-schematic theory standing on the classical logic $\boldsymbol{L} \boldsymbol{K}$ can be regarded as an axiom-schematic theory standing on the intuitionistic logic $\boldsymbol{L} \boldsymbol{J}$ by merely regarding the Peirce rule as an axiom schema. However, we can not regard axiomatic theories standing on $\boldsymbol{L} \boldsymbol{K}$ as axiomatic theories standing on $\boldsymbol{L} \boldsymbol{J}$ in general. In my paper [3], I have described my idea to characterize every axiomatic theory standing on $\boldsymbol{L} \boldsymbol{J}$ 
or $\boldsymbol{L} \boldsymbol{K}$ by a certain relation or by a certain proposition in the primitive logic LO, although we have to reformulate the theory because of a misreasoning in it. (See [2].)

To any axiomatic theory, we associate a predicate or a proposition $\mathfrak{T}$ in $\boldsymbol{L} \boldsymbol{O}$ according as the axiomatic theory stands on $\boldsymbol{L} \boldsymbol{J}$ or $\boldsymbol{L} \boldsymbol{K}$, respectively. With respect to the predicate or proposition $\mathfrak{T}$ in $\boldsymbol{L} \boldsymbol{O}$, we define for any proposition $\mathfrak{P}$ in the axiomatic theory its $\mathfrak{I}$-transform $\mathfrak{P}[\mathbb{I}]$ in $\boldsymbol{L O}$. Then, we have the following theorem: To any axiomatic theory standing on $\boldsymbol{L} \boldsymbol{J}$ or on $\boldsymbol{L} \boldsymbol{K}$, we can find out such a predicate $\mathfrak{T}$ or such a proposition $\mathfrak{T}$, respectively, that any proposition $\mathfrak{B}$ is provable in the axiomatic theory if and only if the $\mathfrak{T}$ transform $\mathfrak{P}^{[\mathfrak{E}]}$ of the proposition $\mathfrak{B}$ is provable in the logic LO. This theorem has been essentially suggested already in [2], but shall be completely proved in the present paper.

The above theorem seems also to lead us to a new understanding of formal theories. Every axiomatic theory characterized by its logic and its axioms would possibly be characterized by a single relation $\mathfrak{T}$ (including the case of a proposition regarding it as a 0 -ary relation $)^{1}$. Possibly, we would have no need to change our logic case by case. This is really the case for axiomatic theories standing on $\boldsymbol{J}$ - or $\boldsymbol{K}$-series logics. It turns out that it is better to regard any relation $\mathfrak{T}$ in $\boldsymbol{L O}$ (i.e. expressible in terms of the logical constants "implication" and "universal quantification" only) as characterizing a formal theory.

For formal theories, we are mostly concerned with what is provable rather than what is true. By means of any relation $\mathfrak{T}$ in $\boldsymbol{L O}$, we can define for every proposition $\mathfrak{P}$ (possibly containing logical constants other than "implication" and "universal quantification") its $\mathfrak{T}$-transform $\mathfrak{P}[\mathfrak{T}]$. Provability in the formal system characterized by $\mathfrak{T}$ can be defined as follows : $\mathfrak{P}$ is called provable in the formal system characterized by $\mathfrak{T}$ if and only if $\mathfrak{\Re}[\mathfrak{T}]$ is provable in the logic $\boldsymbol{L O}$.

To justify this provability notion, we would have to check that any proposition deducible from propositions which are provable in this sense is surely

1) In the present paper, I use the word "relation" in a generalized sense that it denotes any well-formed proposition containing a certain number of parameters. It is called $n$-any $(n$ placed) if we regard it as a relation with respect to variables, say $x_{1}, \cdots, x_{n}$. It is not necessary for any relation with respect to the variables $x_{1}, \cdots, x_{n}$ that every variable $x_{i}$ really occurs in the relation, nor that no variable other than $x_{1}, \cdots, x_{n}$ occurs in the relation. 0-ary relations are occasionally called propositions and unary relations are occasionally called predicates. 
provable in the same sense. In fact, we can prove that this is really the case.

Speaking more exactly, we can assert the following: For any proposition $\mathfrak{I}$ (i.e. $a^{\cdot} 0$-ary relation), any proposition which is tautologically provable in the classical logic $\boldsymbol{L} \boldsymbol{K}$, is provable in this sense, and any proposition which is deducible in $\boldsymbol{L} \boldsymbol{K}$ from propositions which are provable in this sense, is really provable in the same sense. For any relation $\mathfrak{I}$, any proposition which is tautologically provable in the intuitionistic logic $\boldsymbol{L J}$, is provable in this sense, and any proposition which is deducible in $\boldsymbol{L} \boldsymbol{J}$ from propositions which are provable in this sense, is really provable in the same sense. Here we understand that $\mathfrak{I}$ (in both cases) is expressible in terms of "implication" and "universal quantification" only.

Thus, we can introduce a formal theory by means of any relation $\mathfrak{T}$ expressible in terms of "implication" and "universal quantification" only. The formal theory introduced by $\mathfrak{T}$ turns out to be trivial when $(x) \cdots(z) \mathfrak{T}(x, \cdots, z)$ is provable in $\boldsymbol{L} \boldsymbol{O}$. The formal theory can be duly called contradictory in this case. I will denote by [T] the formal theory introduced by the relation $\mathfrak{T}$, and $\mathrm{I}$ will call $\mathfrak{T}$ a taboo relation of the formal theory $[\mathfrak{T}]$. I will also call any formal theory tabooistic if and only if it can be characterized by a relation. According to this terminology, we can say that any axiomatic theory standing on any one of $\boldsymbol{J}$ - or $\boldsymbol{K}$-series logics is tabooistic.

In any tabooistic theory [T] , logical constants other than that of $\boldsymbol{L O}$ can be regarded as defined in connection with the taboo relation $\mathfrak{T}$ for $\mathfrak{T}$-transforms of elementary formulas. This could be regarded as another aspect of tabooistic theories.

To any axiom-schematic theories standing on any one of $\boldsymbol{J}$ - or $\boldsymbol{K}$-series logics, we can associate a predicate schema $\boldsymbol{S}$ or a proposition schema $\boldsymbol{S}$, respectively, in such a way that any proposition $\mathfrak{P}$ is provable in the axiom-schematic theory if and only if $\mathfrak{P}_{[\mathfrak{T}]}$ is provable in $\boldsymbol{L O}$ for some $\mathfrak{T}$ belonging to the schema $\boldsymbol{S}$. Accordingly, we are apt to define further a formal theory by means of any relation schema $\boldsymbol{S}$ by the following stipulation: Any proposition $\mathfrak{P}$ is called provable in the theory if and only if $\mathfrak{B}^{[\mathfrak{I}]}$ is provable in $\boldsymbol{L O}$ for some relation $\mathfrak{T}$ belonging to the relation schema $\boldsymbol{S}$. However, this can not be done generally. For example, we can not assert for every relation schema $\boldsymbol{S}$ in general, that we can find out a relation $\mathfrak{T}$ belonging to the schema $S$ and satisfying $(\mathfrak{P} \wedge \mathfrak{D})^{[\mathfrak{T}]}$ whenever there are a pair of relations $\mathfrak{T}_{1}$ and $\mathfrak{I}_{2}$ belonging to the schema $\boldsymbol{S}$ and satisfying $\mathfrak{B}^{\left[\mathfrak{I}_{1}\right]}$ and $\mathfrak{D}^{\left[\mathfrak{T}_{2}\right]}$. 
Hence, we can define a formal theory by means of a relation schema $\boldsymbol{S}$ only when it is certain that any proposition which is deducible from propositions which are provable in the above sense, is surely provable in the same sense. In this case, the formal theory introduced by the relation schema $\boldsymbol{S}$ is denoted by $[\boldsymbol{S}]$ and the relation schema $\boldsymbol{S}$ is called a taboo schema of the formal theory $[\boldsymbol{S}]$. Any formal theory characterized by a taboo schema is called taboo-schematic. As shown later, any axiom-schematic theory standing on any one of $\boldsymbol{J}$ - or $\boldsymbol{K}$-series logics is taboo-schematic.

It must be natural to ask whether the notions "tabooistic theory" and "taboo-schematic theory" are really more extensive than the notions "axiomatic theory" and axiom-schematic theory," respectively. I believe that this is an important and also interesting problem, even though I can not say anything definite at present.

\section{(1) Preparatory Remarks}

At first, I will give a short sketch of some results in my works [1] and [2].

Let $\mathfrak{T}(x)$ be any $n$-ary relation $(n \geq 0)$. Then, we define $\mathfrak{P}^{\mathfrak{T}}, \mathfrak{P} \wedge_{\mathfrak{T}} \mathfrak{d}$ $\mathfrak{P} \underset{\mathfrak{L}}{\bigvee} \mathfrak{D}, \underset{\mathfrak{x}}{\mathfrak{P}}$, and $(\underset{\mathfrak{x}}{\exists} t) \mathfrak{P}(t)$ for any proposition $\mathfrak{P}$, $\mathfrak{D}$, and $\mathfrak{P}(u)$ as follows :

$$
\begin{aligned}
& \mathfrak{P}^{\mathfrak{T}} \equiv(x)((\mathfrak{P} \rightarrow \mathfrak{T}(x)) \rightarrow \mathfrak{T}(x)), \\
& \mathfrak{P} \underset{\mathfrak{T}}{\wedge} \mathfrak{D} \equiv(x)((\mathfrak{P} \rightarrow(\mathfrak{D} \rightarrow \mathfrak{T}(x))) \rightarrow \mathfrak{T}(x)), \\
& \mathfrak{P} \underset{\mathfrak{T}}{\vee} \mathfrak{D} \equiv(x)((\mathfrak{P} \rightarrow \mathfrak{T}(x)) \rightarrow((\mathfrak{D} \rightarrow \mathfrak{T}(x)) \rightarrow \mathfrak{T}(x))), \\
& \overrightarrow{\mathfrak{T}} \mathfrak{\mathfrak { P }} \equiv \mathfrak{P} \rightarrow(x) \mathfrak{T}(x), \\
& (\underset{\mathfrak{T}}{(\exists} t) \mathfrak{P}(t) \equiv(x)((t)(\mathfrak{P}(t) \rightarrow \mathfrak{T}(x)) \rightarrow \mathfrak{T}(x)),
\end{aligned}
$$

where $x$ stands for a sequence of mutually distinct $n$ variables not occurring free in $\mathfrak{P}$, $\mathfrak{D}$, and $\mathfrak{P}(t)$.

$\mathfrak{P}^{\mathfrak{T}}$ is called the $\mathfrak{T}$-closure of $\mathfrak{P} . \quad \bigwedge_{\mathfrak{T}}, \underset{\mathfrak{T}}{\bigvee_{\mathfrak{T}}}, \underset{\mathfrak{T}}{\mathfrak{T}}$, and $(\underset{\mathfrak{T}}{\mathfrak{G}})$ are called $\mathfrak{T}$ conjunction, $\mathfrak{I}$-disjunction, $\mathfrak{T}$-negation, and $\mathfrak{T}$-existential quantification, respectively.

$\mathfrak{B}^{\mathfrak{T}}$ is always deducible from $\mathfrak{B} . \quad \mathfrak{B}$ is called $\mathfrak{T}$-closed if and only if $\mathfrak{B}$ is deducible from $\mathfrak{P}^{\mathfrak{T}}$. $\mathfrak{P}^{\mathfrak{T}}$ is always $\mathfrak{T}$-closed. $\mathfrak{P} \rightarrow \mathfrak{D}$ is $\mathfrak{T}$-closed if $\mathfrak{D}$ is $\mathfrak{T}$-closed. $(t) \mathfrak{P}(t)$ is $\mathfrak{T}$-closed if $\mathfrak{P}(t)$ is $\mathfrak{T}$-closed for any variable $t$ whatever. $\mathfrak{P} \bigwedge_{\mathfrak{T}} \mathfrak{D}$, $\mathfrak{P} \underset{\mathfrak{T}}{\bigvee} \mathfrak{D}, \underset{\mathfrak{T}}{\mathfrak{P}}$, and $(\underset{\mathfrak{T}}{\exists} t) \mathfrak{B}(t)$ are all $\mathfrak{T}$-closed.

Implication, universal quantification, $\mathfrak{T}$-conjunction, $\mathfrak{T}$-disjunction, $\mathfrak{T}$-negation, and $\mathfrak{T}$-existential quantification for $\mathfrak{T}$-closed propositions satisfy all the inference rules for implication, universal quantification, conjunction, disjunction, negation, and existential 
quantification of $\boldsymbol{L J}$. They satisfy further the Peirce rule if $\mathfrak{T}$ is 0-ary, i.e., $a$ proposition.

Now, we define $\mathfrak{T}$-transform $\mathfrak{P}[\mathfrak{T}]$ of any proposition $\mathfrak{F}$ recursively as follows :

$$
\begin{aligned}
& \mathfrak{P}^{[\mathfrak{T}]} \equiv \mathfrak{P}^{\mathfrak{T}} \quad \text { for any elementary proposition } \mathfrak{P} \text {, } \\
& (\mathfrak{P} \rightarrow \mathfrak{D})^{[\mathfrak{T}]} \equiv \mathfrak{P}[\mathfrak{T}] \rightarrow \mathfrak{Q}[\mathfrak{T}], \\
& \left.((t) \mathfrak{B}(t))^{[\mathfrak{T}]} \equiv(t)(\mathfrak{P}(t))^{[\mathfrak{T}]}\right), \\
& (\mathfrak{P} \wedge \mathfrak{D})^{[\mathfrak{T}]} \equiv \mathfrak{P}^{[\mathfrak{T}]} \widehat{\mathbb{T}}_{\mathfrak{T}} \mathfrak{\mathbb { L }}[\mathfrak{\mathbb { T }}],
\end{aligned}
$$

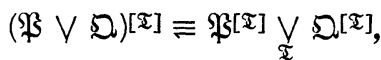

$$
\begin{aligned}
& (\rightarrow \mathfrak{P})^{[\mathfrak{T}]} \equiv \mathfrak{\mathfrak { T }}(\mathfrak{P}[\mathbb{\mathscr { T }}]), \\
& ((\exists t) \mathfrak{P}(t))^{[\mathfrak{I}]} \equiv(\underset{\mathfrak{I}}{\exists} t)\left(\mathfrak{P}(t)^{[\mathfrak{T}]}\right) .
\end{aligned}
$$

Evidently, $\mathfrak{P}[\mathfrak{T}]$ is always $\mathfrak{T}$-closed. $\mathfrak{P}[\mathfrak{T}]$ is expressible in terms of the logical constants "implication" and "universal quantification" only, if $\mathfrak{T}$ is so.

In [2], we had the following theorem : Let $\mathfrak{P}$ be any proposition and let $R$ be any predicate (or proposition) symbol which does not occur in the proposition $\mathfrak{P}$. Then, $\mathfrak{B}$ is provable in $\boldsymbol{L J}$ (or in $\boldsymbol{L} \boldsymbol{K}$ ) if and only if $\mathfrak{B}[R]$ is provable in $\boldsymbol{L O}$.

\section{(2) Tabooistic theories and Taboo-Schematic theories}

Let $\mathfrak{T}$ be any relation expressible in terms of two logical constants "implication" and "universal quantification." Under the "tabooistic theory," we understand the formal theory having the set of logical constants "implication," "universal quantification," "conjunction," "disjunction," "negation," and "existential quantification" in which any proposition $\mathfrak{P}$ is called provable if and only if $\mathfrak{P}^{[\mathfrak{T}]}$ is provable in LO. Any tabooistic theory [T] is called $n$-ary if $\mathfrak{T}$ is an $n$-ary relation.

We can easily prove the following theorems :

Theorem 1. In any tabooistic theory, all the inference rules of $\boldsymbol{L} \boldsymbol{J}$ hold.

THEOREM 2. In any 0-ary tabooistic theory, all the inference rules of $\boldsymbol{L} \boldsymbol{K}$ hold.

Next, let $\boldsymbol{S}$ be any schema (or class) of relations expressible in terms of "implication" and "universal quantification," which satisfies the following condition : For any pair of relations $\mathfrak{T}_{1}$ and $\mathfrak{T}_{2}$ belonging to $\boldsymbol{S}$, there is such a 
relation $\mathfrak{T}$ belonging to $\boldsymbol{S}$ that, for any proposition $\mathfrak{H}, \mathfrak{P}[\mathfrak{x}]$ is provable in $\boldsymbol{L O}$ in so far as either of $\mathfrak{P}^{\left[\mathfrak{x}_{1}\right]}$ or $\left.\mathfrak{\mathfrak { S }} \mathfrak{\mathfrak { x }}_{2}\right]$ is provable.

Under the taboo-schematic theory $[\boldsymbol{S}]$, we understand the formal theory having the logical constants "implication," "universal quantification," "conjunction," "disjunction," "negation" and "existential quantification" in which any proposition $\mathfrak{P}$ is called provable if and only if there is a relation $\mathfrak{T}$ belonging to the schema $\boldsymbol{S}$ which makes $\mathfrak{P}[\mathfrak{I}]$ provable in LO. Any taboo-schematic theory $[\boldsymbol{S}]$ is called $n$-ary if every relation $\mathfrak{T}$ of $\boldsymbol{S}$ is an $n$-ary relation.

We can prove the following theorems:

THeORem 3. In any taboo-schematic theory, all the inference rules of $\boldsymbol{L} \boldsymbol{J}$ hold.

Proof. To prove this theorem, we have only to check the following inference rules of $\boldsymbol{L} \boldsymbol{J}$ :

I. $\mathfrak{A}$ is deducible from $\mathfrak{B}$ and $\mathfrak{B} \rightarrow \mathfrak{A}$.

$\boldsymbol{C}^{*}$. $\quad \mathfrak{A} \wedge \mathfrak{B}$ is deducible from $\mathfrak{A}$ and $\mathfrak{B}$.

D. $\mathfrak{A}$ is deducible from $\mathfrak{B} \vee \mathfrak{C}, \mathfrak{B} \rightarrow \mathfrak{A}$, and $\mathfrak{\mathfrak { C }} \rightarrow \mathfrak{A}$,

I will check here, for example, the inference rule $I$. Namely, let us assume $\mathfrak{B}^{\left[\mathfrak{T}_{1}\right]}$ and $(\mathfrak{B} \rightarrow \mathfrak{A})^{\left[\mathfrak{T}_{2}\right]}$ in $\boldsymbol{L O}$ for two relations $\mathfrak{T}_{1}$ and $\mathfrak{T}_{2}$ of $\boldsymbol{S}$. Then, by assumption for $\boldsymbol{S}$, there is another relation $\mathfrak{T}$ in $\boldsymbol{S}$, for which $\mathfrak{B}[\mathfrak{T}]$ and $(\mathfrak{B} \rightarrow \mathfrak{A})^{[\mathfrak{T}]}$, i.e., $\mathfrak{B}^{[\mathfrak{T}]} \rightarrow \mathfrak{U}[\mathfrak{T}]$ are provable in $\boldsymbol{L O}$ if $\mathfrak{B}^{\left[\mathfrak{T}_{1}\right]}$ and $(\mathfrak{B} \rightarrow \mathfrak{A})^{\left[\mathfrak{X}_{2}\right]}$ are provable. Accordingly, we have $\mathfrak{A}[\mathfrak{x}]$ in $\boldsymbol{L} \boldsymbol{O}$ if we assume $\mathfrak{B}^{\left[\mathfrak{T}_{1}\right]}$ and $(\mathfrak{B} \rightarrow \mathfrak{A})^{\left[\mathfrak{T}_{2}\right]}$ in $\boldsymbol{L} \boldsymbol{O}$.

We can check the inference rules $\boldsymbol{C}^{*}$ and $\boldsymbol{D}$ similarly.

The following theorems can be proved also.

THEOREM 4. In any 0-ary taboo-schematic theory, all the inference rules of $\boldsymbol{L} \boldsymbol{K}$ hold.

In reality, it is rather hard to develop tabooistic theories or tabooschematic theories in $\boldsymbol{L O}$. Theorems $1-4$ suggest a practical way of developing tabooistic theories or taboo-schematic theories by means of inference rules of $\boldsymbol{L} \boldsymbol{J}$ (and of $\boldsymbol{L} \boldsymbol{K}$ for 0-ary tabooistic theories as well as for 0-ary taboo-schematic theories) starting from some provable propositions. 


\section{(3) Axiomatic theories standing on $J$ - or $K$-series logics}

Theorems in this chapter can be regarded as a reformulation of the main result of my paper [3].

In the following, throughout this chapter, we deal with axiomatic theories having the axiom system $\mathfrak{A}_{1}, \cdots, \mathfrak{A}_{n}$ and standing on any one of $\boldsymbol{J}$ - or $\boldsymbol{K}^{-}$ series logics. $\mathfrak{A}_{1} \wedge \cdots \wedge \mathfrak{A}_{n}$ is denoted simply by $\mathfrak{A}$. The symbol $R^{2)}$ denotes a predicate (for $\boldsymbol{J}$-series logics) or a proposition (for $\boldsymbol{K}$-series logics) which is not a primitive notion of the respective axiomatic theory. I will define the predicate $\mathfrak{T}$ (for $\boldsymbol{J}$-series logics) or the proposition $\mathfrak{I}$ (for $\boldsymbol{K}$-series logics) by

$$
\begin{aligned}
& \mathfrak{T}(x) \equiv \mathfrak{A}[R] \rightarrow R(x) \\
& (\mathfrak{I} \equiv \mathfrak{U}[R] \rightarrow R) .
\end{aligned}
$$

Theorem 5. Any axiomatic theory standing on the logic $\boldsymbol{L} \boldsymbol{J}$ is equivalent to the unary tabooistic theory [?]

Proof. Any proposition $\mathfrak{F}$ is provable in the axiomatic theory if and only if $\mathfrak{A} \rightarrow \mathfrak{B}$ is provable in $\boldsymbol{L} \boldsymbol{J}$. On the other hand, according to the conclusion of my paper [2], the proposition $\mathfrak{A} \rightarrow \mathfrak{B}$ is provable in $\boldsymbol{L} \boldsymbol{J}$ if and only if

$$
(\mathfrak{A} \rightarrow \mathfrak{H})^{[R],} \quad \text { i.e., } \quad \mathfrak{O}[R] \rightarrow \mathfrak{\$}[R]
$$

is provable in $\boldsymbol{L} \boldsymbol{O}$.

Now, I assert that $\mathfrak{2}[R] \rightarrow \mathfrak{B}^{[R]}$ is tautologically equivalent to $\mathfrak{P}[\mathbb{2}]$ in $\boldsymbol{L O}$.

To show this, I will prove at first that $\mathfrak{X}[R] \rightarrow \mathfrak{B}[R]$ is equivalent to $\mathfrak{A}[R] \rightarrow \mathfrak{B}[\mathfrak{T}]$ in LO . For, by definition, the predicate $\mathfrak{T}$ is equivalent to the predicate $R$ under the assumption $\mathfrak{A}[R]$. Hence, $\mathfrak{S}[\mathfrak{L}]$ must be also equivalent to $\mathfrak{B}[R]$ under the same assumption. Consequently, $\mathfrak{A}[R] \rightarrow \mathfrak{P}[R]$ is equivalent to $\mathfrak{A}[R] \rightarrow \mathfrak{P}[\mathbb{L}]$ in $\boldsymbol{L} \boldsymbol{O}$.

I will prove next that $\mathfrak{P}[\mathfrak{T}]$ is equivalent to $\mathfrak{A}[R] \rightarrow \mathfrak{F}^{[\mathfrak{I}]}$ in $\boldsymbol{L} \boldsymbol{O}$. For, the $\mathfrak{T}$-transform $\mathfrak{P}[\mathfrak{x}]$ of $\mathfrak{P}$ is $\mathfrak{I}$-closed as has been remarked. Hence, $\mathfrak{P}[\mathfrak{T}]$ is tautologically equivalent to

$$
\begin{array}{ll} 
& (x)((\mathfrak{P}[\mathfrak{T}] \rightarrow \mathfrak{T}(x)) \rightarrow \mathfrak{T}(x)), \\
\text { i.e., } & (x)((\mathfrak{P}[\mathfrak{T}] \rightarrow \mathfrak{T}(x)) \rightarrow(\mathfrak{X}[R] \rightarrow R(x)) .
\end{array}
$$

2) $R(x)$ (or $R$ ) is an elementary proposition if $R$ is a predicate (or a proposition) symbol. 
The last proposition is evidently equivalent to

$$
\begin{array}{ll} 
& \mathfrak{N}[R] \rightarrow(x)((\mathfrak{F}[\mathfrak{T}] \rightarrow \mathfrak{T}(x)) \rightarrow(\mathfrak{N}[R] \rightarrow R(x))), \\
\text { i.e., } & \mathfrak{U}[R] \rightarrow(x)((\mathfrak{P}[\mathfrak{T}] \rightarrow \mathfrak{T}(x)) \rightarrow \mathfrak{T}(x)) .
\end{array}
$$

Because $\mathfrak{P}^{[\mathfrak{I}]}$ is $\mathfrak{T}$-closed, this proposition is equivalent to $\mathfrak{U}[R] \rightarrow \mathfrak{P}[\mathfrak{I}]$. Thus, $\mathfrak{P}[\mathfrak{T}]$ is proved to be equivalent to $\mathfrak{A}[R] \rightarrow \mathfrak{P}[\mathfrak{T}]$ in $\boldsymbol{L} \boldsymbol{O}$.

Since we have proved in $\boldsymbol{L} \boldsymbol{O}$ that $\mathfrak{A}[R] \rightarrow \mathfrak{B}[R]$ is equivalent to $\mathfrak{A}[R] \rightarrow \mathfrak{P}[\mathscr{\complement}]$ and that $\mathfrak{A}[R] \rightarrow \mathfrak{P}[\mathfrak{T}]$ is equivalent to $\mathfrak{P}[\mathfrak{T}]$, we see that $\mathfrak{A}[R] \rightarrow \mathfrak{P}[R]$ is equivalent to $\mathfrak{S}[\mathfrak{T}]$ in $\boldsymbol{L O}$.

Hence, any proposition $\mathfrak{P}$ is provable in the axiomatic theory standing on $\boldsymbol{L} \boldsymbol{J}$ if and only if $\mathfrak{\Re}[\mathfrak{T}]$ is provable in $\boldsymbol{L} \boldsymbol{O}$. So, $\mathfrak{P}$ is provable in the axiomatic theory standing on $\boldsymbol{L} \boldsymbol{J}$ if and only if it is provable in the tabooistic theory [? $]$. In other words, the axiomatic theory standing on the logic $\boldsymbol{L} \boldsymbol{J}$ is equivalent to the tabooistic theory [T].

Theorem 6. Any axiomatic theory standing on the logic $\boldsymbol{L} \boldsymbol{K}$ is equivalent to the 0-ary tabooistic theory [T].

We can prove this theorem quite similarly as the proof of Theorem 5 .

Because $\boldsymbol{L P}$ and $\boldsymbol{L Q}$ are sub-logics of $\boldsymbol{L} \boldsymbol{J}$ and $\boldsymbol{L} \boldsymbol{K}$, respectively, we have also the following theorems :

Theorem 7. Any axiomatic theory standing on $\boldsymbol{L P}$ is equivalent to a unary tabooistic theory without negation as its logical constant.

Theorem 8. Any axiomatic theory standing on $\boldsymbol{L Q}$ is equivalent to a 0-ary tabooistic theory without negation as its logical constant.

Since $\boldsymbol{L} \boldsymbol{M}$ and $\boldsymbol{L} \boldsymbol{N}$ can be regarded as a special case of $\boldsymbol{L P}$ and $\boldsymbol{L Q}$ having the propositional constant $\hat{\Lambda}$ as a primitive notion, we have the following theorems :

Theorem 9. Any axiomatic theory standing on $\boldsymbol{L} \boldsymbol{M}$ is equivalent to a unary tabooistic theory when we regard negation as no logical constant but regard $\rightarrow \mathfrak{S}$ as standing for $\mathfrak{B} \rightarrow \boldsymbol{\Lambda}$ for a proposition constant $\curlywedge$.

TheOREM 10. Any axiomatic theory standing on $\boldsymbol{L N}$ is equivalent to $\dot{a}$ 0-ary tabooistic theory when we regard negation as no logical constant but regard $\rightarrow \mathfrak{P}$ as standing for $\mathfrak{\Re} \rightarrow$ ᄉ for a proposition constant $\curlywedge$. 
Remark 1. Any axiomatic theory standing on any sub-logic $\boldsymbol{L}$ of $\boldsymbol{L} \boldsymbol{J}$ (or $\boldsymbol{L} \boldsymbol{K}$ ) can be also regarded as unary (0-ary) tabooistic even when $\boldsymbol{L}$ does not have "conjunction" but it does have "implication" and "universal quantification" as its logical constants. We can modulate the proof of Theorem 5 (Theorem 6) as follows :

Let $\mathfrak{A}_{1}, \cdots, \mathfrak{A}_{n}$ be an axiom system of any axiomatic theory standing on $\boldsymbol{L}$, and let $R$ be any predicate symbol (proposition symbol) which is not a primitive notion of the axiomatic theory. Then, we have only to define a taboo predicate $\mathfrak{I}$ (taboo proposition $\mathfrak{I}$ ) by

$$
\begin{aligned}
& \mathfrak{T}(x) \equiv \mathfrak{U}_{1}^{[R]} \rightarrow\left(\mathfrak{U}[R] \rightarrow\left(\cdots \rightarrow\left(\mathfrak{U}_{n}[R] \rightarrow R(x)\right) \cdots\right)\right) \\
& \left(\mathfrak{T} \equiv \mathfrak{U}_{1}^{[R]} \rightarrow\left(\mathfrak{U}_{2}[R] \rightarrow\left(\cdots \rightarrow\left(\mathfrak{U}_{n}[R] \rightarrow R\right) \cdots\right)\right)\right) .
\end{aligned}
$$

This taboo predicate $\mathfrak{T}$ (taboo proposition $\mathfrak{T}$ ) can be proved to be equivalent to $\mathfrak{A}[R] \rightarrow R(x)$ (equivalent to $\mathfrak{A}[R] \rightarrow R$ ) if $\boldsymbol{L}$ has the logical constant $\wedge$ and $\mathfrak{A}$ stands for $\mathfrak{A}_{1} \wedge \cdots \wedge \mathfrak{A}_{n}$.

We can follow the whole proof of Theorem 5 (Theorem 6) for the $\operatorname{logic} \boldsymbol{L}$ and for the taboo predicate $\mathfrak{T}$ (taboo proposition $\mathfrak{T}$ ) even though some expressions occurring in the proof become somewhat cumbersome.

Remark 2. Theorems 5-10 as a whole can be regarded as a generalization of the main result of my work [2]. Namely, any one of $\boldsymbol{J}$ - or $\boldsymbol{K}$ series logics can be regarded as an axiomatic theory having an empty axiom system and standing on the logic in question itself. In this case, the taboo predicate or the taboo proposition of the corresponding tabooistic theory turns out to be the predicate symbol $R$ or the proposition symbol $R$, respectively.

\section{(4) Axiom-schematic theories standing on $J$ - or $K$-series logics}

For axiom-schematic theories standing on $\boldsymbol{J}$ - or $\boldsymbol{K}$-series logics, the following theorems hold :

Theorem 11. Any axiom-schematic theory standing on the logic $\boldsymbol{L J}$ is equivalent to a unary taboo-schematic theory.

Proof. Let $\boldsymbol{C}$ be the axiom-schema of any axiom-schematic theory standing on the logic $\boldsymbol{L} \boldsymbol{J}$, and let $R$ be a predicate symbol which is not a primitive notion of the axiom-schematic theory. Further, let $\boldsymbol{S}$ be the 
schema of predicates of the form $\mathfrak{X}^{[R]} \rightarrow R(x)$, where $\mathfrak{A}$ stands for $\mathfrak{A}_{1} \wedge \cdots \wedge \mathfrak{A}_{n}$ for axioms $\mathfrak{A}_{i}$ belonging to $\boldsymbol{C}, n$ being any number including 0 . Then, we can define a taboo-schematic theory $[\boldsymbol{S}]$ by means of the predicate schema $\boldsymbol{S}$ regarding it as a schema of taboo predicates. The unary taboo-schematic theory $[\boldsymbol{S}]$ is equivalent to the axiom-schematic theory having $\boldsymbol{C}$ as its axiom schema.

Namely, I will show at first that we can define a taboo-schematic theory by means of $\boldsymbol{S}$. To show this, let us take any pair of predicates $\mathfrak{T}_{1}$ and $\mathfrak{I}_{2}$ belonging to $\boldsymbol{S}$. Then, $\mathfrak{I}_{1}(x)$ and $\mathfrak{T}_{2}(x)$ have the forms $\mathfrak{A}_{1}^{[R]} \rightarrow R(x)$ and $\mathfrak{V}_{2}{ }^{[R]} \rightarrow R(x)$ for $\mathfrak{A}_{1}$ and $\mathfrak{A}_{2}$ belonging to $\boldsymbol{C}$ by definition of the predicate schema $\boldsymbol{S}$. Now, let us define a new predicate $\mathfrak{T}(x)$ by

$$
\mathfrak{T}(x) \equiv\left(\mathfrak{A}_{1} \wedge \mathfrak{A}_{2}\right)^{[R]} \rightarrow R(x) .
$$

For this predicate $\mathfrak{I}, \mathrm{I}$ will prove the following: For any proposition $\mathfrak{P}$, the $\mathfrak{T}$-transform $\mathfrak{P}[\mathfrak{T}]$ of $\mathfrak{S}$ is provable in $\boldsymbol{L O}$ if either the $\mathfrak{I}_{1}$-transform $\mathfrak{P}^{\left[\mathfrak{T}_{1}\right]}$ or the $\mathfrak{T}_{2}$-transform $\mathfrak{P}_{\left[\mathfrak{T}_{2}\right]}$ of $\mathfrak{P}$ is provable in LO. To show this, assume that $\mathfrak{P}_{\left[\mathfrak{x}_{t}\right]}$ $(i=1,2)$ is provable in $\boldsymbol{L} \boldsymbol{O}$. Then, according to Theorem 5, the proposition $\mathfrak{F}$ is provable in the axiomatic theory having $\mathfrak{A}_{i}$ as its axiom and standing on the logic $\boldsymbol{L} \boldsymbol{J}$. In other words, $\mathfrak{A}_{i}$ implies $\mathfrak{F}$ in $\boldsymbol{L} \boldsymbol{J}$. Because $\mathfrak{A}_{1} \wedge \mathfrak{A}_{2}$ implies $\mathfrak{A}_{i}$ in the same logic, the proposition $\mathfrak{F}$ is also provable in the axiomatic theory having $\mathfrak{A}_{1} \wedge \mathfrak{A}_{2}$ as its axiom. Accordingly, $\mathfrak{P}[\mathbb{x}]$ is provable in $\boldsymbol{L} \boldsymbol{O}$ by virtue of Theorem 5 .

Now, by Theorems 3 and 5, we can easily check that the given axiomschematic theory is equivalent to the taboo-schematic theory $[\boldsymbol{S}]$.

Theorem 12. Any axiom-schematic theory standing on the logic $\boldsymbol{L} \boldsymbol{K}$ is equivalent to a 0-ary taboo-schematic theory.

Just as the case of axiomatic theories standing on the logics $\boldsymbol{L P}, \boldsymbol{L Q}$, $\boldsymbol{L M}$, or $\boldsymbol{L} \boldsymbol{N}$, we have the following theorems for axiom-schematic theories standing on these logics.

THEOREM 13. Any axiom-schematic theory standing on $\boldsymbol{L P}$ is equivalent to a unary taboo-schematic theory without negation as its logical constant.

THeOREm 14. Any axiom-schematic theory standing on $\boldsymbol{L Q}$ is equivalent to a 0-ary taboo-schematic theory without negation as its logical constant. 
Theorem 15. Any axiom-schematic theory standing on $\boldsymbol{L M}$ is equivalent to a unary tabooistic theory when we do not regard negation as a logical constant but regard $\rightarrow \mathfrak{B}$ as standing for $\mathfrak{\mathfrak { S }} \rightarrow \boldsymbol{\wedge}$ for a proposition constant $\wedge$.

Theorem 16. Any axiom-schematic theory standing on $\boldsymbol{L} \boldsymbol{N}$ is equivalent to a 0-ary taboo-schematic theory when we do not regard negation as a logical constant but regard $\rightarrow \mathfrak{B}$ as standing for $\mathfrak{\$} \rightarrow \wedge$ for a proposition constant $\curlywedge$.

\section{REFERENCES}

[1] Ono, K.: On universal character of the primitive logic, Nagoya Math. J., 27 (1966), 331-353.

[2] —_: Reduction of logics to the primitive logic, J. of Math. Soc. Jap., 19-3 (1967), 384-398.

[ 3 ] — : Taboo versus axiom, Nagoya Math. J., 28 (1966), 73-77.

Mathematical Institute,

Nagoya University. 